\title{
Disfunción tiroidea posexposición a medio de contraste yodado
}

\author{
Cristian Iván García Rincón ${ }^{1}$; Luz Yaneth Becerra Salazar²; Germán Alberto Moreno Gómez ${ }^{3}$; Henry Mauricio \\ Arenas Quintero ${ }^{4}$
}

${ }^{1}$ Médico especialista en Medicina Interna. Universidad Tecnológica de Pereira, Risaralda. Hospital Pablo Tobón Uribe, Medellín, Antioquia.

${ }^{2}$ Médica General. Hospital Departamental Santa Sofía de Caldas. Manizales, Caldas.

${ }^{3}$ Médico Magister en Epidemiología Clínica. Docente Titular del Programa de Medicina Universidad Tecnológica de Pereira. Pereira, Risaralda.

${ }^{4}$ Médico Endocrinólogo de la Clínica Comfamiliar Risaralda. Miembro de Número Asociación Colombiana de Endocrinología, Diabetes y Metabolismo. Docente de Cátedra Posgrado Medicina Interna. Universidad Tecnológica de Pereira. Pereira, Risaralda

Conflictos de intereses: los autores de este artículo declaran no tener conflicto de intereses

\section{Resumen}

$\mathrm{L}$ a exposición a cantidades excesivas de yodo bloquea la actividad de la enzima peroxidasa tiroidea (TPO), un fenómeno conocido como efecto de Wolff-Chaikoff. Clínicamente, éste puede llevar a hipotiroidismo. Por el contrario, cuando un tirocito contiene valores bajos de yodo y es expuesto a elevadas concentraciones del mismo, aumenta de forma significativa la producción de hormona tiroidea, llevando frecuentemente a hipertiroidismo clínico y bioquímico, fenómeno conocido como efecto de Jod-Basedow. Tanto el hiper como el hipotiroidismo aumentan el riesgo de enfermedad arterial coronaria, miopatía ventricular izquierda, anormalidades electrofisiológicas, además de incrementar la mortalidad cardiovascular y por todas las causas.

Palabras clave: Disfunción tiroidea, medio de contraste yodado, hipertiroidismo, hipotiroidismo.

\footnotetext{
Abstract

Exposure to excessive amounts of iodine blocks the activity of the enzyme thyroid peroxidase (TPO), a phenomenon known as Wolf-Chaikoff effect. Clinically, this effect can lead to hypothyroidism. On the other hand, when thyrocites have low iodine levels and are exposed to a high iodine concentration, they increase significantly their production of thyroid hormone thus leading to hyperthyroidism, a phenomenon known as Jod-Basedow effect. Both hypothyroidism and hyperthyroidism increase the risk of coronary artery disease, left cardiomyopathy, electrophysiological abnormalities, and all-cause mortality.

Key words: Thyroid dysfunction, iodine contrast medium, hyperthyroidism, hypothyroidism.
}

\section{Introducción}

Existen dos principales tipos de hormona tiroidea: tiroxina (T4) y triyodotironina (T3). Bajo circunstancias normales, cerca del $90 \%$ de la producción tiroidea se hace en la forma de T4 y 10\% como T3. Los tirocitos concentran yodo contra gradiente. Para la síntesis de hormona tiroidea se requiere de un mecanismo molecular complejo en el cual el NIS (cotransportador yoduro/sodio) juega un papel clave ${ }^{(1)}$. El NIS es una proteína transportadora de la membrana basolateral del tirocito que tiene la habilidad para concentrar yoduro en estas células, en concentraciones incluso hasta 20-40 veces mayores a la concentración sérica. Ha sido estudiado intensamente, ya que es un factor crítico en muchos estados patológicos. Una vez en los tirocitos, el yoduro es organificado en residuos de tirosina presentes en la tiroglobulina ( $\mathrm{Tg}$ ). Este proceso tiene lugar en la membrana apical de los tirocitos. La enzima peroxidasa tiroidea (TPO) - una selenoproteína- es clave en este proceso, junto con el peróxido de hidrógeno ${ }^{(1)}$.

El yodo es un elemento esencial en la síntesis de hormonas tiroideas. La ingesta diaria recomendada para adultos es de alrededor de $150 \mathrm{mcg}$. La glándula tiroides tiene mecanismos intrínsecos regulatorios que mantienen la función tiroidea, incluso en la presencia de exceso de yodo. Cuando se administran grandes cantidades de yodo a personas con función tiroidea normal, la síntesis de las hormonas tiroideas disminuye de forma transitoria durante al menos dos días. Este efecto inhibitorio agudo es denominado de Wolff-Chaikoff, y se presenta como consecuencia de la mayor concentración de yodo ${ }^{(2)}$.

Los medios de contraste yodados (MCY) son agentes farmacológicos administrados de forma rutinaria. Durante los últimos 20 años su uso se ha aumentado de forma dramática, en paralelo con un aumento de 4 a 8 veces en el empleo de tomografías computarizadas (TC) contrastadas y angiografías coronarias $^{(3)}$. Aunque se conocen complicaciones asociadas al uso de medios de contraste, los estudios se han enfocado principalmente hacia la nefropatía inducida por medio de contraste, mientras las alteraciones a nivel de la función tiroidea han sido relegadas a un segundo plano ${ }^{(3,4)}$. Los medios de contraste yodados hidrosolubles, en los pacientes con función renal normal, son casi completamente eliminados del cuerpo en las 24 
horas subsecuentes a la inyección. En aquellos pacientes con una tasa de filtración glomerular disminuida, la eliminación es retardada. Sin embargo, no hay evidencia de un riesgo mayor de tirotoxicosis en pacientes con función renal severamente disminuida (tasa de filtración glomerular (TFG) menor de 20 $\mathrm{ml} / \mathrm{min})^{(2)}$.

Las regulaciones de control de calidad, realizadas a los medios de contraste yodados, toleran pequeñas cantidades de impureza bajo la forma de yodo libre (hasta $50 \mathrm{mg} / \mathrm{mL}$ ), en medios de contraste hidrosolubles. La concentración de yodo libre es bastante menor a la cantidad de yodo unido orgánicamente al anillo de benzeno y su efecto a nivel del metabolismo de la tiroides ha sido considerado insignificante. No obstante, el uso de MCY no es recomendado en pacientes con enfermedad de Graves, bocio multinodular y autonomía tiroidea, debido al riesgo de tirotoxicosis. Se ha considerado que ni el hipotiroidismo, ni reducciones en T3 - síndrome de T3 bajo-, requieren precauciones especiales, pese a que incluso se han descrito casos de hipotiroidismo subclínico transitorio después de la inyección de $\mathrm{MCY}^{(5)}$.

En pacientes con patología cardiaca, la conversión periférica reducida de T4 a T3, constituye un síndrome de T3 bajo, hallazgo frecuente principalmente en pacientes con falla cardiaca, infarto agudo de miocardio y después de cirugía cardiaca.

Tanto el hipotiroidismo como el síndrome de T3 bajo son reportados como predictores independientes de pronóstico adverso, no sólo en la población general, sino también en los pacientes con patología cardiaca y están asociados a un aumento de eventos cardiovasculares duros, así como a mayor mortalidad general ${ }^{(6,7)}$. Esto debería tenerse en consideración a la hora de realizar estudios imaginológicos con medios de contraste yodados en pacientes con patología cardiovascular o riesgo de la misma.

\section{Disfunción tirodea y medio de contraste yodado}

Una dosis típica de MCY contiene aproximadamente $13.500 \mathrm{mg}$ de yodo libre y 15-60 gramos de yodo unido, el cual puede ser liberado como yodo libre en el organismo. Esto representa una carga de yodo aguda entre 90 y cientos de veces mayor a la ingesta de yodo recomendada diariamente (150 $\mathrm{mcg}$ ). El exceso de yodo libre en la sangre (ingerido o inyectado) puede causar tirotoxicosis en pacientes en riesgo ${ }^{(2,3,8)}$.

Pese al aumento en el uso de MCY y a una asociación biológicamente plausible entre su administración y la presencia de disfunción tiroidea (hiper o hipotiroidismo), ha habido pocos estudios al respecto ${ }^{(3)}$, arrojando evidencia limitada, fundamentalmente por: tamaño de muestras insuficiente, no distinción entre hiper o hipotiroidismo, no restricción a casos de enfermedad tiroidea incidente, seguimiento por corto término y, principalmente, ausencia de grupo control frente a los cuales comparar el riesgo de disfunción tiroidea asociada a la administración de $\mathrm{MCY}^{(3)}$.
La exposición a cantidades excesivas de yodo bloquea la actividad de la TPO, fenómeno de Wolff-Chaikoff ${ }^{(9)}$, se presenta cuando se produce un aumento brusco en las concentraciones plasmáticas de yodo (como sucede tras la inyección intravenosa de contraste yodado), y aumenta el yodo dentro de la glándula tiroides por encima de un determinado nivel, haciendo que se disminuya la organificación de este elemento, se aumente la relación monoyodotironina/diyodotironina y por ende se disminuye la síntesis de hormonas tiroideas. Clínicamente, éste puede llevar a hipotiroidismo. En condiciones normales, las concentraciones de hormonas tiroideas circulantes permanecen dentro del rango eutiroideo y en pocos días, se recupera la síntesis hormonal (fenómeno de escape) (2). Sin embargo, en los pacientes con enfermedad tiroidea (enfermedad de Graves, tiroiditis de Hashimoto, etc.) o incluso en aquellos quienes presentan disfunción tiroidea subclíni$\mathrm{ca}^{(9)}$, estos mecanismos de adaptación a menudo fracasan, las sobrecargas de yodo pueden desencadenar hipotiroidismo y más raramente, hipertiroidismo. El exceso en el consumo de yodo también reduce la liberación de T4 y T3 desde la glándula tiroides. Esto resulta en pequeñas disminuciones séricas de T3 y T4, con aumentos compensatorios de las concentraciones de TRH y TSH ${ }^{(2,10)}$. Estos eventos son característicamente observados en los tirocitos llenos de yodo.

La reducción de la T3 libre (T3L) después de la inyección de MCY puede ser explicada por dos mecanismos: por la respuesta tiroidea inhibitoria a la sobrecarga de yodo y por la reducción periférica de la deyodación de T4. En el escenario de la angiografía coronaria, el aumento en las concentraciones séricas de T4 libre (T4L) puede estar relacionado con el desplazamiento de la unión de la T4 a la albúmina, provocado esto por la administración intravenosa y el uso concomitante de heparina durante el cateterismo cardiaco. El aumento de la T4L puede también representar un mecanismo de retroalimentación, comprometiendo el hipotálamo ${ }^{(5)}$.

Por el contrario, cuando un tirocito tiene valores de yodo muy bajos y es expuesto a éste, aumenta de forma significativa la producción de hormona tiroidea, llevando frecuentemente a hipertiroidismo clínico y bioquímico, fenómeno conocido como efecto de Jod-Basedow ${ }^{(1,11)}$. Es poco claro el mecanismo subyacente a este fenómeno, pero se presume que pueda ser debido a una rápida yodación de la tiroglobulina (Tg) pobremente yodada, o por efectos de un tejido tiroideo con funcionamiento autónomo subyacente ${ }^{(1)}$.

En condiciones fisiológicas normales, la captura y organificación de yoduro y la síntesis subsecuente y liberación de T3 y T4 son reguladas de forma estrecha. No obstante, después de la exposición a niveles suprafisiológicos, se puede sobrepasar la capacidad regulatoria, precipitando hipertiroidismo por vía Jod-Basedow. Este efecto se ha observado con 300-500 mg de yodo, cantidad mucho menor a la dosis que se administra con el medio de contraste. El efecto de Jod-Basedow es clásicamente descrito entre aquellos pacientes que tienen enfermedad autoinmune tiroidea, en quienes la deficiencia de yodo enmas- 
cara la expresión del hipertiroidismo, en pacientes con bocios nodulares autónomos y en poblaciones geriátricas (en quienes la prevalencia de enfermedad tiroidea nodular es más alta). Aunque controversial, la tirotoxicosis inducida por yodo ha sido reportada en pacientes sin enfermedad tiroidea subyacente ${ }^{(3)}$.

El hipertiroidismo inducido por yodo no es una entidad de etiología simple; puede ocurrir en pacientes con una variedad de enfermedades tiroideas subyacentes, dentro de las cuales las más importantes son: enfermedad de Graves y bocio multinodular, especialmente en ancianos y en quienes viven en áreas con deficiencia de yodo. La incidencia de hipertiroidismo inducida por yodo en áreas deficientes ha sido estimada alrededor de 1,7\%, mientras en áreas con suficiencia de yodo, la incidencia es mucho más baja. Muchos de los pacientes que presentan tirotoxicosis en este contexto tienen enfermedad tiroidea multinodular y la mayoría son clínicamente eutiroi$\operatorname{deos}^{(2,10)}$. Los pacientes en alto riesgo deberían ser cuidadosamente monitorizados por los clínicos, después de la exposición a medio de contraste yodado, sin embargo, la profilaxis en este grupo no se recomienda de forma rutinaria ${ }^{(12,13)}$.

\section{Estudios clínicos de disfunción tiroidea inducidos por me- dio de contraste:}

Existen pocos estudios al respecto. Las poblaciones en estudio y los resultados pueden diferir dependiendo de si el estudio fue realizado en un área con deficiencia o suficiencia de yodo.

Así, por ejemplo, en áreas sin deficiencia de yodo ${ }^{(2)}$, un estudio no mostró efectos en los niveles séricos de T3 y T4L hasta 56 días después de la angiografía coronaria y administración de meglumina ioxaglato ${ }^{(14)}$. De otro lado, siete pacientes con bocio multinodular de una cohorte de 24.600 TC realizadas en un periodo de tres años, requirieron admisión hospitalaria por hipertiroidismo severo inducido por medio de contraste, posterior a la administración de 3-12 mg de yodo libre en medio de contraste no iónico ${ }^{(15)}$. Después de la realización de TC de tiroides, usando $100 \mathrm{~mL}$ de iohexol, 8 de 22 pacientes con enfermedad tiroidea presentaron un cambio temporal en la función tiroidea ${ }^{(16)}$. En poblaciones geriátricas australianas, la tirotoxicosis inducida por yodo después de la administración de iopamidol, $370 \mathrm{mg} / \mathrm{dL}$, para imágenes con contraste, fue la causa de 7 de 28 casos de hipertiroidismo observados durante un periodo de 20 meses $^{(17)}$. En otro estudio del mismo grupo, en 60 pacientes mayores de 70 años con hipertiroidismo, 23\% estuvieron expuestos a medio de contraste yodado en los seis meses previos a la evaluación ${ }^{(18)}$.

Los estudios en áreas deficientes de yodo también han sido escasos. Así, la prevalencia y patogénesis de tirotoxicosis después de la administración de medio de contraste fue evaluada entre 1971 y $1979^{(19)}$, encontrando que en 89 (15\%) de 663 pacientes con tirotoxicosis, la condición podría ser relacionada con la administración del medio de contraste yodado (el 95\% de los casos sucedió después de 12 semanas). En 63\% de los pacientes hubo bocio subyacente y la mayoría de estos pacientes fueron ancianos. En otro estudio de pacientes no aleatoriza- dos, sólo 2 de 788 presentaron hipertiroidismo en las siguientes 12 semanas de la coronariografía ${ }^{(20)}$. Por el contrario, en otro estudio, la administración de medio de contraste yodado no iónico a 102 pacientes eutiroideos no produjo hipertiroidismo en paciente alguno, a pesar del gran número de pacientes con bocio y nodularidades tiroideas detectadas previamente a la administración del medio de contraste; este mismo estudio mostró que la morfología tiroidea por ecografía, no fue un factor pronóstico para el desarrollo de hipertiroidismo.

En 1996, Hehrmann et al. ${ }^{(21)}$ reportaron que después de 21 días de administración de grandes dosis de medio de contraste existe una pequeña disminución, seguida por un aumento en los límites normales en T4L, y una disminución, seguida por un incremento rápido (menos de cinco días), en los límites normales de TSH. Más recientemente, Fassbender et al. ${ }^{(22)}$ estudiaron 102 pacientes sometidos a angiografía coronaria, hallando en el análisis de subgrupos un pequeño aumento de los niveles de TSH en aquellos pacientes con glándulas tiroides pequeñas, pero valores séricos disminuidos en aquellos pacientes con glándulas tiroides aumentadas de tamaño. Adicionalmente, observaron un aumento discreto en los niveles de T4L en aquellos pacientes con glándulas tiroides aumentadas de tamaño y niveles de TSH normales bajos.

En 2004, Gartner y Weiseel(23) realizaron un estudio con 22 pacientes a quienes evaluaron temprano después de la administración de medio de contraste yodado, hallando un aumento en los niveles de TSH tres a cinco días después de la administración del medio de contraste, con aumentos por fuera del límite superior de la normalidad en alrededor del 18\% en aquellos pacientes con valores basales de TSH normales-altos, sin evidenciar alteraciones en la medición de los valores séricos de las hormonas tiroideas (T3 o T4L). Lo anterior, en el contexto del estudio, sugiere la posibilidad de hipotiroidismo subclínico transitorio, condición vista más frecuentemente en aquellos pacientes con tiroiditis autoinmune (de Hashimoto) ${ }^{(24)}$. Así, a la luz de los resultados del estudio, en muchos pacientes eutiroideos no se observan cambios en los parámetros funcionales tiroideos, aunque se pueden observar casos de hiper o hipotiroidismo transitorios. Es necesario tener en cuenta que la administración de medio de contraste a poblaciones geriátricas puede conducir a hipertiroidismo subclínico de larga duración, con aumento de los niveles de T4L y disminución de la TSH sérica por periodos tan largos como ocho semanas luego de la inyección, lo cual se presume que pueda estar favorecido por nódulos autónomos no diagnosticados en las glándulas tiroides de estos pacientes.

En 2008, Mekaru et al. ${ }^{(25)}$ publicaron un estudio en el que evaluaron el efecto sobre la función tiroidea, de un medio de contraste yodado liposoluble (lipiodol), empleado en histerosalpingografía en pacientes sometidas a estudio de infertilidad entre 1996 y 2006, en una institución de salud japonesa, a quienes se les medía TSH y T4L antes y varios meses después del procedimiento. En total, se estudiaron 214 pacientes, divididas en tres grupos con base en los valores iniciales: 180 
eutiroideas, $28 \%$ hipotiroidismo subclínico y 13\% hipertiroidismo subclínico. El número de pacientes en el grupo de hipotiroidismo subclínico que desarrollaron hipotiroidismo después del procedimiento (36\%) fue significativamente mayor que las que lo hicieron en el grupo de eutiroideas $(2,2 \%)$. Adicionalmente, tres de los pacientes inicialmente con hipotiroidismo subclínico requirieron terapia con remplazo hormonal tiroideo durante su seguimiento, sugiriendo la necesidad de evaluación de perfil de hormonas tiroideas antes y después de procedimientos con MCY en este tipo de pacientes.

En enero de 2012, Rhee et al. ${ }^{(3)}$ publicaron el primer gran estudio controlado de la asociación entre exposición a medio de contraste y la incidencia de enfermedad funcional tiroidea, observando una asociación significativa entre la exposición al medio de contraste e hipertiroidismo incidente, hiper e hipotiroidismo manifiesto incidente, pero no hipotiroidismo incidente en general. La asociación observada entre la exposición al medio de contraste y el hipertiroidismo incidente es explicada, probablemente, por las cargas de yoduro incluidas en el medio de contraste.

Independiente del mecanismo, la asociación observada entre la exposición al medio de contraste yodado y el hipertiroidismo incidente es de importancia clínica considerable, dados los efectos que el hipertiroidismo prolongado tiene sobre el sistema cardiovascular y en términos de supervivencia ${ }^{(3,6,7,26)}$.

El exceso de yodo también puede llevar a hipotiroidismo a través de la alteración del transportador de sodio yoduro, de la organificación del yodo y de la síntesis y secreción de hormona tiroidea mediante el efecto de Wolff-Chaikoff. En el estudio de Rhee et al., no se observó asociación entre exposición a medio de contraste e hipotiroidismo incidente en general, incluyéndose como potenciales explicaciones un error tipo II, la ausencia de un efecto biológico o el encubrimiento de un efecto fisiológico transitorio debido al intervalo de tiempo prolongado entre la exposición al medio de contraste y el seguimiento en las medidas de $\mathrm{TSH}^{(3)}$. Además, posterior a la presentación del efecto de WolffChaikoff, los pacientes frecuentemente recuperan la función tiroidea normal sobre semanas a meses, lo cual puede ser la explicación plausible de la asociación existente entre la exposición al medio de contraste y el hipotiroidismo incidente observado cuando el intervalo entre las medidas basales y de seguimiento se realizan a corto tiempo pero no en largos periodos ${ }^{(18)}$.

En 2013, Paolo Marraccine et al. ${ }^{(5)}$ publicaron un estudio en el que evaluaron la prevalencia de disfunción tiroidea en aquellos pacientes sometidos a la administración de medio de contraste yodado para la realización de coronariografía, hallando que: 1). La concentración de yodo libre en cinco soluciones comerciales de medio de contraste yodado se encontró por debajo de los límites tóxicos (de daño) establecidos (hasta $50 \mathrm{mg} / \mathrm{mL}$ ) por las entidades de regulación del control de calidad; pese a ello, observaron un aumento inesperado en la concentración de yodo urinario después de la administración del medio de contraste, el cual no puede ser explicado exclusivamente por el yodo libre administrado en la solución, arguyendo como explicación razonable, la de- yodación del medio de contraste yodado circulante. 2). Adicionalmente, estos autores documentan en su serie de 1.752 pacientes, disfunción tiroidea previa a la administración del medio de contraste yodado en cerca del $40 \%$ de la población en estudio, encontrando el síndrome T3 bajo en $28,1 \%$ e hipotiroidismo en $10,3 \%$ de la población, documentando que, durante un seguimiento de 63,5 meses en promedio. Estas dos entidades se convierten en factores de riesgo independientes para eventos cardiovasculares mayores, así como para mortalidad total. De forma simultánea, los autores encontraron que $1,9 \%$ de los pacientes presentaban hipertiroidismo; este bajo porcentaje explicable por la no inclusión de muchos de los pacientes con hipertiroidismo subyacente, ante el riesgo de tirotoxicosis, además de la premedicación con metimazol, yodo radioactivo y perclorato de potasio. 3). De manera concomitante, los autores observaron que la administración de medio de contraste puede adicionalmente modificar el perfil de las hormonas tiroideas; en particular, los pacientes con síndrome de T3 bajo tuvieron una reducción significativa en T3 libre (T3L) sérica, 48 horas después de la coronariografía, encontrándose además cambios en los valores de T4L y TSH ${ }^{(5)}$.

En ese mismo año, Ozcan et al. publicaron un estudio observacional en el cual evaluaron 101 pacientes eutiroideos quienes fueron programados para angiografía coronaria, hallando en el seguimiento que los valores de TSH estuvieron bajos en $6,9 \%$ de ellos a las cuatro semanas y en 5,9\% a las ocho semanas, con descensos estadísticamente significativos, sin cambios en los valores de T4L y T3, sugiriendo con ello la asociación de hipertiroidismo subclínico con la administración de MCY en pacientes sometidos a cateterismo cardiaco $^{(27)}$.

La incidencia de hipotiroidismo postangiografía encontrada en los niños menores de 3 años en una evaluación realizada por Del Cerro Marín et al. en 2000, justificaría en principio la realización de un control de función tiroidea tras dicha exploración. La presencia de múltiples malformaciones asociadas a cardiopatía es el factor de riesgo más claramente relacionado, tanto al desarrollo de hipotiroidismo como a la mayor duración de éste. Puesto que las situaciones de hipotiroidismo de duración mayor de tres semanas se produjeron sólo en los portadores de múltiples malformaciones, los autores sugieren que, al menos en este tipo de pacientes, debería ser obligada la tamización de función tiroidea tras la angiografía con contraste yodado, ya que algunos de estos niños pueden precisar tratamiento sustitutivo ${ }^{(28)}$.

En términos generales, existen dos mecanismos fisiológicos implicados en la disfunción tiroidea posterior a la administración de MCY: efecto de Wolff-Chaikoff y efecto de Jod-Basedow. Con relación al hipertiroidismo inducido por yodo, se consideran como potenciales poblaciones en riesgo: pacientes que vivan en áreas con deficiencia de yodo, ancianos y antecedentes de bocio nodular o difuso no tóxico. Por el contrario, las poblaciones que habitan en áreas con suficiencia de yodo, tienen antecedentes de tiroidectomía parcial, enfermedad de Graves tratada, tiroiditis subaguda, posparto o de Hashimoto, podrían ser susceptibles de desarrollar hipotiroidismo inducido por yodo ${ }^{(29)}$ 
Tabla 1. Disfunción tiroidea y medio de contraste yodado

\begin{tabular}{|l|l|l}
\hline Disfunción tiroidea & Hipotiroidismo & Hipertiroidismo \\
\hline $\begin{array}{l}\text { Mecanismo } \\
\text { fisiopatológico } \\
\text { propuesto }\end{array}$ & $\begin{array}{l}\text { Falla en el escape al } \\
\text { efecto Wolff-Chaikoff }\end{array}$ & Efecto de Jod-Basedow \\
\hline Yodo en el área & Usualmente suficiente & Usualmente insuficiente \\
\hline $\begin{array}{l}\text { Potencial población en } \\
\text { riesgo }\end{array}$ & $\begin{array}{l}\text { Tiroidectomía parcial } \\
\text { Enfermedad de Graves } \\
\text { tratada } \\
\text { Tiroiditis subaguda o } \\
\text { posparto } \\
\text { Tiroiditis de Hashimoto }\end{array}$ & $\begin{array}{l}\text { Ancianos } \\
\text { Bocio nodular } \\
\text { Bocio difuso no tóxico } \\
\text { Enfermedad de Graves }\end{array}$ \\
\hline Prevalencia & $\begin{array}{l}\text { Variable, pocos } \\
\text { estudios. } \\
\text { Desconocida en nuestro } \\
\text { medio }\end{array}$ & $\begin{array}{l}\text { Variable, pocos } \\
\text { estudios. } \\
\text { Desconocida en nuestro } \\
\text { medio }\end{array}$ \\
\hline
\end{tabular}

(ver tabla 1). Tanto el hiper como el hipotiroidismo aumentan el riesgo de enfermedad arterial coronaria, miopatía ventricular izquierda, anormalidades electrofisiológicas, además de mortalidad cardiovascular y por todas las causas ${ }^{(3,6,7,26)}$.

Los datos de los estudios anteriores refuerzan la necesidad de una evaluación concienzuda de los pacientes que serán sometidos a la administración de medio de contraste yodado, dada la posibilidad, en el caso de cateterismo cardiaco, de hallar pacientes con disfunción tiroidea establecida hasta en el $40 \%$ de los casos y en el contexto de imágenes tomo y urográficas, la posibilidad de condicionar alteraciones en el perfil de hormonas tiroideas de dichos pacientes.

\section{Conclusiones}

La exposición súbita a altas cargas de yodo puede alterar la regulación hormonal tiroidea, resultando en hipotiroidismo (efecto de Wolff-Chaikoff) o hipertiroidismo (efecto de JodBasedow) como se ha explicado previamente. La disfunción tiroidea inducida por medio de contraste yodado es rara. Se ha preconizado previamente que la inyección de medio de contraste no afecta las pruebas de función tiroidea en pacientes con tiroides normal. Sin embargo, esta presunción debería ser reconsiderada a partir de los resultados de estudios realizados en los últimos años.

En este contexto y, dado el uso frecuente de los medios de contraste yodados, en la práctica son necesarios más estudios dirigidos a establecer asociaciones, relaciones de causalidad y a aclarar los potenciales mecanismos fisiopatológicos subyacentes, con el fin de instaurar medidas dirigidas a prevenir la disfunción tiroidea posterior a la administración de medios de contraste yodados.

Aunque en la actualidad no contamos con guías para la disfunción tiroidea posterior a exposición a medios de contraste yodados, sugerimos la realización de pruebas de función tiroidea en mayores de 65 años, niños y mujeres que deseen fertilidad, que vayan a ser llevados a estudios con contraste o ya hayan sido expuestos a medios de contraste yodados, por las implicaciones clínicas que la disfunción tiroidea tiene en estos grupos especiales.

\section{Referencias}

1. Stathatos N. Thyroid Physiology. Medical Clinics of North America 2012;96(2):165-173.

2. Van der Molen AJ, Thomsen HS, Morcos SK. Effect of iodinated contrast media on thyroid function in adults. Eur Radiol 2004;14(5):902-907.

3. Rhee C.M, Bhan I, Alexander E.K, Brunelli S.M. Association between iodinated contrast media exposure and incident hyperthyroidism and hypothyroidism. Archives of Internal Medicine 2012;172(2): 153-159.

4. Katzberg RW, Haller C. Contrast-induced nephrotoxicity: clinical landscape. Kidney Int Suppl 2006;100:S3-S7.

5. Marraccini P, Bianchi M, Bottoni A, Mazzarisi A, Coceani M, Molinaro S, Lorenzoni V, Landi P y lervasi G. Prevalence of thyroid dysfunction and effect of contrast medium on thyroid metabolism in cardiac patients undergoing coronary angiography. Acta radiol 2012;54(1):42-7.

6. Haentjens P, Van Meerhaegue A, Poppe K, Velkeniers B. Subclinical thyroid dysfunction and mortality: an estimate of relative and absolute excess all-cause mortality based on tie-to-event data from cohort studies. Eur J Endocrinol 2008;159(3): 329-341.

7. Sgarbi JA, Matsumura LK, Kasamatsu TS, Ferreira SR y Maciel RMB. Subclinical thyroid dysfunctions are independent risk factors for mortality in a 7.5- year followup: The Japanese-Brazilian thyroid study. Eur J Endocrinol 2010;162(3): 569-77.

8. Moisey RS, McPherson S, Wright M, Orme SM. Thyroiditis and iodide mumps following an angioplasty. Nephrol Dial Transplant 2007;22(4):1250-1252

9. Almandoz JP y Gharib H. Hypothyroidismo: etiology, Diagnosis, and management. Med Clin North Am 2012;96(2):203-221.

10. Roti E, Uberti ED. Iodine excess and hyperthyroidism. Thyroid 2001;11(5):493500 .

11. Woeber KA. Iodine and thyroid disease. Med Clin North Am 1991;75(1):169-78.

12. Van der Molen AJ. Effect on thyroid function. Medical Radiology 2009;139-146.

13. Hintze G, Bolmbach O, Fink H, Burkhardt U y Kobberling J. Risk of iodine-induced thyrotoxicosis after coronary angiography: an investigation in 788 unselected subjects. Eur J Endocrinol 1999;140(3):264-267.

14. Grainger RG, Pennington GW. A study of the effect of sodium/meglumine ioxaglate (Hexabrix) on thyroid function. Br J Radiol 1981;54(645):768-772

15. De Bruin TJW. Iodide induced hyperthyroidism with computed tomography con-

trast fluids. Lancet 1994; 343(8906):1160-1161.

16. Nygaard B, Nygaard T, Jensen LI et al. Iohexol: effects on uptake of radioactive iodine in the thyroid and on thyroid function. Acad Radiol 1998;5(6):409-414.

17. Martin FI, Tress BW, Colman PG, Deam DR. Iodine-induced hy-perthyroidism due to non-ionic contrast radiography in the elderly. Am J Med 1993;95(1):78-82.

18. Martin FI, Deam DR. Hyperthyroidism in elderly hospitalized patients. Clinical features and treatment outcomes. Med J Aust 1996;164(4):200-203.

19. Stiedle B. Iodine-induced hyper-thyroidism after contrast media. Animal experimental and clinical studies. Fortschr Geb Rontgenstrahlen Nuklearmed Erganzugsbd 1989;128:6-14.

20. Hintze G, Blombach 0, Fink H, Burkhardt U, Köbberling J. Risk of iodine-induced thyrotoxicosis after coronary angiography: an investigation in 788 unselected subjects. Eur J Endocrinol 1999;140(3):264-267.

21. Hehrmann R, Klein D, Mayer D et al. Hyperthyreoserisiko bei Kontrastmitteluntersuchungen. Aktuel Radiol 1996;6:243-248.

22. Fassbender WJ, Vogel C, Doppl W et al. Thyroid function, thyroid immunoglobulin status, and urinary iodine excretion after enteral contrast-agent administration by endoscopic ret-rograde cholangiopancreatography. Endoscopy 2001;33(3):245-252.

23. Gartner W, Weissel M. Do iodine-containing contrast media induce clinically relevant changes in thyroid function parameters of euthyroid patients within the first week? Thyroid 2004;14(7):521-4.

24. Roberts CG, Ladenson PW. Hypothyroidism. Lancet 2004;363(9411):793-803.

25. Mekaru K, Kamiyama S, Masamoto H, Sakumoto K, Aoki Y. Thyroid function after hysterosalpingography using an oil-soluble iodinated contrast medium. Gynecol Endocrinol. 2008;24(9):498-501.

26. Seigel SC, Hodak SP. Thyrotoxicosis. Med Clin North Am 2012;96(2):175-201.

27. Ozkan $\mathrm{S}$, et al. Thyroid functions after contrast agent administration for coronary angiography: a prospective observational study in euthyroid patients. Anadolu Kardiyol Derg. 2013;13:363-369.

28. Del Cerro Marín J, et al. Alteraciones de la función tiroidea en niños con cardiopatía congénita tras la realización de cateterismo con contraste yodado. Rev Esp cardio 2000;53:517-24

29. Pearce EN. Iodine-induced thyroid dysfunction: comment on "association between iodinated contrast media exposure and incident hyperthyroidism and hypothyroidism". Arch Intern Med. 2012 Jan 23;172(2):159-61. 